\title{
Effects of dihydrotestosterone on synaptic plasticity of the hippocampus in mild cognitive impairment male SAMP8 mice
}

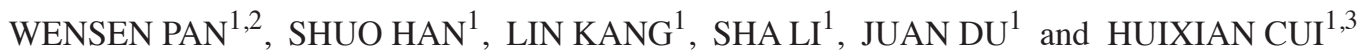 \\ ${ }^{1}$ Department of Human Anatomy, Hebei Medical University, Shijiazhuang, Hebei 050017; ${ }^{2}$ Department of \\ Respiration Medicine, The Second Hospital of Hebei Medical University, Shijiazhuang, Hebei 050000; ${ }^{3}$ Hebei \\ Key Laboratory for Brain Aging and Cognitive Neuroscience, Shijiazhuang, Hebei 050031, P.R. China
}

Received April 3, 2016; Accepted June 21, 2016

DOI: $10.3892 / \mathrm{etm} .2016 .3470$

\begin{abstract}
The current study focused on how dihydrotestosterone (DHT) regulates synaptic plasticity in the hippocampus of mild cognitive impairment male senescence-accelerated mouse prone 8 (SAMP8) mice. Five-month-old SAMP8 mice were divided into the control, castrated and castrated-DHT groups, in which the mice were castrated and treated with physiological doses of DHT for a period of 2 months. To determine the regulatory mechanisms of DHT in the cognitive capacity, the effects of DHT on the morphology of the synapse and the expression of synaptic marker proteins in the hippocampus were investigated using immunohistochemistry, qPCR and western blot analysis. The results showed that the expression of cAMP-response element binding protein $(C R E B)$, postsynaptic density protein 95 (PSD95), synaptophysin $(S Y N)$ and developmentally regulated brain protein (Drebrin) was reduced in the castrated group compared to the control group. However, DHT promoted the expression of CREB, PSD 95, SYN and Drebrin in the hippocampus of the castrated-DHT group. Thus, androgen depletion impaired the synaptic plasticity in the hippocampus of SAMP8 and accelerated the development of Alzheimer's disease (AD)-like neuropathology, suggesting that a similar mechanism may underlie the increased risk for AD in men with low testosterone. In addition, DHT regulated synaptic plasticity in the hippocampus of mild cognitive impairment (MCI) SAMP8 mice and delayed the progression of disease to Alzheimer's dementia. In conclusion, androgen-based hormone therapy is a potentially useful strategy for preventing the progression of $\mathrm{MCI}$ in aging men. Androgens enhance synaptic markers (SYN, PSD95, and Drebrin), activate CREB, modulate the fundamental biology of synaptic structure, and lead to the structural changes of plasticity in the hippocampus, all of which result in improved cognitive function.
\end{abstract}

Correspondence to: Dr Huixian Cui, Department of Human Anatomy, Hebei Medical University, 361 Zhongshan East Road, Shijiazhuang, Hebei 050017, P.R. China

E-mail: deep_respiration@sina.com.cn

Key words: Alzheimer's disease, dihydrotestosterone, cognition disorders, mild, aging, mice

\section{Introduction}

An important characteristic of aging is cognitive decline. Previous findings have shown that the decline of cognitive function was associated with reduced plasticity in the hippocampus (1-2). Decreased androgen levels associated with aging have always been considered to be associated with an increased risk of mild cognitive impairment (MCI) and Alzheimer's disease (AD). In 2011, the National Institute on Aging and the Alzheimer's Association proposed new guidelines for AD (1-4). These guidelines recommend that MCI be considered a transitional condition with cognitive changes between normal aging and $\mathrm{AD}(5,6)$. Through evaluation and control of risk factors for conversion from MCI to AD, onset of the disease can be prevented or delayed. The pathophysiology of MCI includes significant neuronal loss in the hippocampus and decreased blood flow in the subiculum (7). Neurofibrillary tangles and $\beta$-amyloid $(\mathrm{A} \beta)$ were observed in individuals with MCI $(8,9)$. Cerebrospinal fluid also shows a disproportionate increase in $\tau$ protein levels relative to individuals from a similar age group (10). Patients with MCI usually have decreased gonadal steroids at the same time.

Depletion of sex steroid hormones with increasing age is the most significant risk factor for the development of AD. Previous findings indicated that estrogen and progesterone (P4) at menopause in women and testosterone in aging men are AD risk factors (11-18). Women have greater vulnerability to AD than men, as observed by relatively sudden and extensive loss of $17 \beta$-estradiol (E2) and P4 at menopause. A decrease of total testosterone $(12,13)$ or free testosterone levels in plasma have been identified in men with AD when compared to patients (15) of vascular dementia and age-matched controls $(11,14,19)$.

Estrogens and androgens can play a protective role against AD-related neurodegeneration $(14,20)$. Men who become hypogonadal in later life often report problems with their memory. Lower than normal testosterone levels have also been detected in patients prior to the onset of $\mathrm{AD}$ and in younger, late-onset, male $\mathrm{AD}$ patients when compared to the appropriate controls. Age-related depletion of testosterone is a risk factor for AD in men (20). Since testosterone is metabolized in the brain into the androgen dihydrotestosterone (DHT) and estrogen E2, testosterone can mediate its effects through 
androgen and/or estrogen pathways. The reduction of DHT in elderly males was negatively associated with the incidence of $\mathrm{AD}$, and it may be able to decrease the activity of the androgen receptor. Previous findings showed that androgen is involved in the development of the central nervous system as well as the maintenance of normal function during maturation (21-23).

In recent decades, the progressive reduction of structural and functional plasticity in hippocampus and the prefrontal cortex were thought to be associated with the gradual decline in cognitive function (24). There was a close associated between synaptic plasticity and sex hormones. It was previously reported that the density of synapses in the hippocampus of adult female rat fluctuates naturally with the levels of ovarian steroids. In adult female rats, the estradiol level in vivo was associated with hippocampal pyramidal cell synapse density and quantity, as well as dendritic spine density (25). The density of the dendritic spines of the pyramidal neurons in the brain regions related to learning and memory, such as the hippocampus, is associated with the estrogen level (26). Thus, the regulatory mechanism of androgens on synaptic plasticity has been considered. Similarly, the density of the dendritic spines of the pyramidal neurons in the hippocampus is also modulated in vivo by the depletion and replacement of androgens (27).

The senescence-accelerated mouse prone 8 (SAMP8) is characterized by an age-related spontaneous deterioration in learning and memory that are similar to AD (28). Previous findings have shown that SAMP8 is a good model of cognitive decline with aging (29-30). Our previous studies (31) identified a fluctuant trend of the density of dendritic spines and, $\mathrm{A} \beta$ deposits, leading to cognitive and neurobiological changes in 5-month-old SAMP8 mice. Thus, 'middle-aged' SAMP8 mice are a suitable model for basic MCI research (31). In the MCI stage of SAMP8 mice, the synaptic injuries have already appeared. As such, the pathological changes of the synapses are a key problem in cognitive dysfunction (32).

Gonadectomy (GDNX) in castrated male rats reduced CA1 spine synapse density compared with the sham-operated controls (32). Treatment of GDNX rats with DHT or testosterone propionate increased spine synapse density to approximately the same levels when compared with intact males. However, an increase in synapse density was not observed in the GDNX animals after treatment with estradiol. These data indicated that androgen is essential for the normal spine synapse density in the CA1 region of the male rat hippocampus. The lack of response to estradiol suggests that testosterone acts directly on hippocampal androgen receptors rather than indirectly via local estrogen biosynthesis (27).

Previous studies conducted on SAMP8 mice identified that the serum testosterone levels quickly decreased in the aging process, with a similar gradual reduction in dendritic spine density and quantity (30-32). The aging core and behavioral experiments were also improved after providing a physiological dose of DHT (1 mg/kg) compared with the castration group (33). Therefore, it is essential to determine the mechanisms of DHT in hippocampal synaptic plasticity.

Postsynaptic density protein 95 (PSD95) in hippocampus exhibited a significant reduction in MCI compared to subjects without dementia. The expression of synaptophysin (SYN), a key player in membrane trafficking events preceding exocytosis that modulates activity-dependent exocytosis, was relatively decreased in castrated aging mice (34). Drebrin is an f-actin postsynaptic binding protein that is associated with synaptic plasticity (35). Previous studies have reported that the levels of Drebrin decreased in the hippocampus of MCI cases, and were associated with the cognitive decline (12). Synaptic plasticity-associated proteins [e.g., SYN, PSD95, developmentally regulated brain protein (Drebrin)] and the memory formation-related protein cAMP-response element binding protein (CREB), were correlated with animal behavior and synaptic morphology (36-39).

The present study focused on whether DHT altered the expression of CREB, PSD95, SYN and Drebrin and examined the protective mechanisms and neurological basis of DHT. In our experiment, immunohistochemistry, western blot analysis and quantitative polymerase chain reaction (qPCR) analysis were used to detect the expression of the aforementioned proteins and to observe the regulation of DHT on synaptic plasticity in the hippocampus of MCI SAMP8 mice. The results confirmed those of our previous study regarding the effects of DHT on the behavior and synaptic plasticity in MCI SAMP8 mice, and provided a valuable theoretical basis for the protective effects of DHT in MCI.

\section{Materials and methods}

Animals and study groups. Five-month-old SAMP8 mice were used as experimental animals ( $\mathrm{n}=45,28-32 \mathrm{~g})$, and randomly divided into the sham-operated control group (P8 group), castration group, and castration plus dihydrotestosterone group (DHT group). Animals in this group received the same surgical castration operation as the castrated group, but without removing the testicles. Mice in the castrated group underwent bilateral orchiectomy. Mice in the DHT group received bilateral orchiectomy and DHT injection. Medicine was administered three days after castration (product code: 2500981; International Laboratory, South San Francisco, CA, USA), for 60 days by subcutaneous injection $(1 \mathrm{mg} / \mathrm{kg}$ per day administered between 5:00 and 6:00 p.m.). Other groups were injected with the same dose of sterile medical corn oil $(0.1 \mathrm{ml})$. SAMP8 male mice were given as gifts by David Yew, Professor of the Chinese University of Hong Kong and bred by our laboratory. For breeding of mice standard rodent diet and water method were used, with a $12 \mathrm{~h}$ light-dark cycle (lights at 6:00 a.m.) and room temperature maintained at $21 \pm 2^{\circ} \mathrm{C}$.

The experimental procedures were implemented in accordance with the use of mammal in neuroscience health guidelines, recognized by the Association of Animal Experimentation Ethics, Hebei Medical University.

Immunohistochemical staining. Each group of mice $(\mathrm{n}=5)$ were anesthetized by $1 \%$ sodium pentobarbital $[80 \mathrm{mg} / \mathrm{kg}$, intraperitoneal (i.p.) injection] and perfused with $0.9 \%$ saline through the left ventricle, and fixed with $4 \%$ paraformaldehyde, $\mathrm{pH}$ 7.4. The brain was carefully removed, specimens were separated between the superior colliculus and optical chiasm, and fixed overnight at $4^{\circ} \mathrm{C}$. The tissue was divided into two halves along the median plane, and the gradient ethanol was dehydrated. The tissue was cleared in xylene and embedded in paraffin. Paraffin blocks were cut into $5 \mu \mathrm{m}$ 
Table I. Primers.

\begin{tabular}{|c|c|c|c|}
\hline Gene & NCBI GeneID & Forward/reverse primer & $\begin{array}{l}\text { Amplicon size } \\
\text { (bp) }\end{array}$ \\
\hline drebrin & 56320 & $\begin{array}{l}\text { 5'-AGGAGCAGTCTATCTTTGGTGA-3' } \\
\text { 5'-CACTGTCCGATGCCTTTATGAA-3' }\end{array}$ & 246 \\
\hline syn & 20977 & $\begin{array}{l}\text { 5'-CAGTTCCGGGTGGTCAAGG-3' } \\
\text { 5'-ACTCTCCGTCTTGTTGGCAC-3' }\end{array}$ & 138 \\
\hline creb & 12912 & $\begin{array}{l}\text { 5'-CAGGGGTCGCAAGGATTGAAG-3' } \\
\text { 5'-ATCGCCTGAGGCAGTGTACT-3' }\end{array}$ & 101 \\
\hline psd95 & 13385 & $\begin{array}{l}\text { 5'-TGAGATCAGTCATAGCAGCTACT-3' } \\
\text { 5'-CTTCCTCCCCTAGCAGGTCC-3' }\end{array}$ & 107 \\
\hline$G A P D H$ & 14433 & $\begin{array}{l}\text { 5'-AGGTCGGTGTGAACGGATTTG-3' } \\
\text { 5'-GGGGTCGTTGATGGCAACA-3' }\end{array}$ & 95 \\
\hline
\end{tabular}

syn, synaptophysin; $p s d 95$, post synaptic density protein 95; creb, cAMP-response element binding protein; drebrin, developmentally regulated brain protein.

serial sections along the sliding microtome (Leica-RM2145; Leica, Mannheim, Germany) in the coronal position. Two consecutive $50 \mu \mathrm{m}$ tissues were collected and placed on polylysine-coated slides to detect the protein expression of CREB, SYN, PSD95 and Drebrin. Following deparaffinization and hydration, the sections were subjected to antigen retrieval using a microwave for $30 \mathrm{~min}$ and immersed in $3 \%$ hydrogen peroxide in methanol for $30 \mathrm{~min}$ to eliminate endogenous peroxidase activity. The sections were incubated with 5\% goat serum to block non-specific binding, followed by overnight incubation with rabbit anti-SYN antibody (1:200, product code: ab14692; Abcam, Cambridge, MA, USA), rabbit anti-PSD95 antibody (1:200, product code: ab12093; Abcam), rabbit anti-CREB antibody (1:200, code: ab32515; Abcam), and rabbit anti-Drebrin antibody (1:100, code: ab60933; Abcam) at $4^{\circ} \mathrm{C}$. After washing with PBS, the sections were incubated with biotinylated goat anti-rabbit IgG for $2 \mathrm{~h}$. A computer image analysis system Image-Pro Plus 6.0 (Media Cybernetics, Inc., Rockville, MD, USA) was used to determine the average values of the optical density (OD) of SYN, PSD95, Drebrin and CREB in the hippocampal CA1 region. Ten sections were analyzed for each mouse, and the average OD value was determined for each mouse.

Western blot analysis. After sample collection, lysis buffer was added, and the samples were homogenized under ice-cooling, and incubated for $30 \mathrm{~min}$. The samples were then centrifuged for $15 \mathrm{~min}$ at $4^{\circ} \mathrm{C}$ at $10,000 \mathrm{x} \mathrm{g}$ and the supernatant was collected. A BAC microplate reader (VersaMax enzyme standard instrument, Molecular Devices, LLC. Sunnyvale CA, USA) was used to determine protein content. Subsequently, $100 \mu 1$ protein was added to an equal volume of $2 \mathrm{X}$ upper sample buffer, and mixed at $95^{\circ} \mathrm{C}$ for $5 \mathrm{~min}$, for denaturation of the protein. The samples were then centrifuged and cooled to room temperature $21 \pm 2^{\circ} \mathrm{C}$. A constant voltage of electrophoresis of upper sample was $80 \mathrm{~V}$. When the sample reached the junction between stacking and separating gel, the constant voltage was adjusted to $120 \mathrm{~V}$, stopping electrophoresis. The constant voltage of the cutting gel and transferring membrane was $300 \mathrm{~V}$, which was stopped after a 60-min wet transfer. Non-fat dry milk (5\%) was used for blocking for $1 \mathrm{~h}$ at room temperature. Then, $1 \mathrm{X}$ TBS was used to dilute SYN, PSD95, Drebrin, CREB antibody at 1:5,000, followed by overnight incubation at $4^{\circ} \mathrm{C}$. Fluorescent secondary antibody was added, and kept in the dark at $37^{\circ} \mathrm{C}$ prior to agitation for $1 \mathrm{~h}$. The Li-Cor Odyssey fluorescence scanning imaging system (Gene Company Limited, Pleasanton, California, USA) was employed to perform semi-quantitative analysis to yield relevant bands. Arbitrary units (AU) (darea • density) were used to represent the area $\mathrm{x}$ gray value of protein bands. GAPDH expression was viewed as an internal reference. The ratio between the target protein and internally referred AU represented the relative protein expression levels of each group.

$q P C R$ analysis. Each group of mice $(\mathrm{n}=5)$ was anesthetized with $1 \%$ sodium pentobarbital ( $80 \mathrm{mg} / \mathrm{kg}$, i.p. injection). RNA samples were extracted using TRIzol reagent from the hippocampus. Ultraviolet NanoDrop lite spectrophotometer (Thermo Fisher Scientific, Waltham, MA, USA) (the A260) was used to measure the amount of RNA. The superscript first-strand synthesis system (Invitrogen Life Technologies, Carlsbad, CA, USA) was used to reverse transcribe $500 \mathrm{ng}$ RNA into complementary DNA. Following transcription, $1 \mu \mathrm{l}$ RT product combined 2.5 units was used to start the volume of Taq DNA polymerase (Sigma-Aldrich, St. Louis, MO, USA) (50 $\mu \mathrm{l}$ buffer contains $20 \mathrm{pmol}$ antisense primer, $10 \mathrm{mM}$ Tris- $\mathrm{HCl}, 50 \mathrm{mM} \mathrm{KCl}, 2.5 \mathrm{mM} \mathrm{MgCl}_{2}$ and $0.2 \mathrm{mM}$ dNTP). Chemically produced primers (Sangon Biotech Co., Ltd., Shanghai, China) are listed in Table I.

The $\mathrm{Eco}^{\mathrm{TM}}$ Real-Time PCR system (Illumina Inc., San Diego, CA, USA) was used to quantitatively determine the relative levels of mRNAs. GAPDH expression was viewed as an internal reference, and the ratio between the data and internal reference indicated the relative level of the target gene. 
Table II. Results of immunohistochemical staining shows the effects of castration and DHT intervention on the conversion from MCI to Alzheimer's dementia in SAMP8 mice. ${ }^{\mathrm{a}}$

\begin{tabular}{lcccc}
\hline Group & CREB & SYN & PSD95 & Drebrin \\
\hline Castration & $0.136 \pm 0.025$ & $0.553 \pm 0.034$ & $0.636 \pm 0.046$ & $0.089 \pm 0.014$ \\
DHT & $0.216 \pm 0.035^{\mathrm{b}}$ & $0.843 \pm 0.107^{\mathrm{b}}$ & $0.935 \pm 0.073^{\mathrm{b}}$ & $0.159 \pm 0.015^{\mathrm{b}}$ \\
P8 & $0.270 \pm 0.046^{\mathrm{b}}$ & $0.887 \pm 0.089^{\mathrm{b}}$ & $0.983 \pm 0.116^{\mathrm{b}}$ & $0.174 \pm 0.018^{\mathrm{b}}$ \\
\hline
\end{tabular}

${ }^{a}$ Immunohistochemical staining detected SYN, PSD95, Drebrin, and CREB gene expression in the Castration, DHT and P8 groups. ${ }^{b} \mathrm{P}<0.01$. DHT, dihydrotestosterone; MCI, mild cognitive impairment; SAMP8, senescence-accelerated mouse prone 8; SYN, synaptophysin; PSD95, postsynaptic density protein 95; CREB, cAMP-response element binding protein; Drebrin, developmentally regulated brain protein.
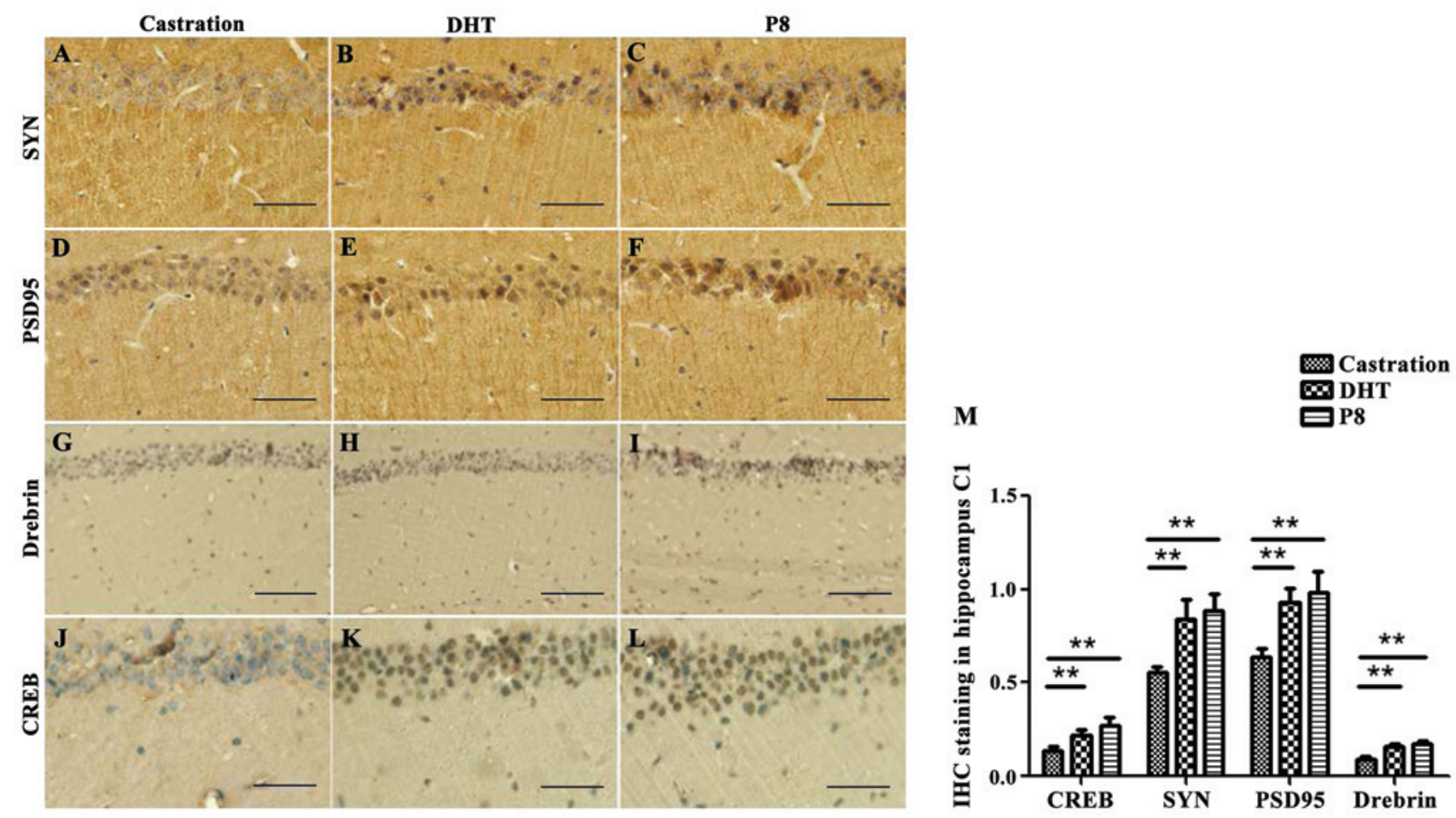

Figure 1. Immunohistochemical staining shows the effects of castration and DHT intervention on the conversion from MCI to Alzheimer's dementia in SAMP8 mice. Immunohistochemical staining detected (A-C) SYN, (D-F) PSD95, (G-I) Drebrin and (J-K) CREB protein expression in the (A, D, G and J) castration, (B, E, H and K) DHT and (C, F, I and L) P8 groups. A statistical graph showing the difference of protein expression in the (M) four groups. The results are presented as the mean \pm standard deviation $(n=5)$. Statistical analysis was performed using one-way ANOVA with LSD post-hoc test. ${ }^{* *} \mathrm{P}<0.01$. DHT, dihydrotestosterone; MCI, mild cognitive impairment; SAMP8, senescence-accelerated mouse prone 8; SYN, synaptophysin; PSD95, postsynaptic density protein 95; CREB, cAMP-response element binding protein; Drebrin, developmentally regulated brain protein.

\section{Results}

Immunohistochemical staining. Immunohistochemical staining showed that the positive products of CREB protein were mainly distributed in the neuronal membrane, cytoplasm and projections. Compared with the castration group, the staining in projections of the P8 and DHT groups was dense and thick, and the cytoplasmic membrane immunoreactive substance increased significantly (Fig. 1A-C). The OD value of the castration group was $0.135 \pm 0.025$, which was significantly lower than that of the $\mathrm{P} 8$ group value of $0.270 \pm 0.046(\mathrm{P}<0.01)$. The OD value of DHT group was $0.216 \pm 0.034$ and was significantly increased compared to the Castration group $(\mathrm{P}<0.01)$.

Immunohistochemical staining for PSD95, SYN and Drebrin showed that brownish yellow granules were located in the neuropil of pyramidal cells (Fig. 1D-L). The expression of the PSD95, SYN and Drebrin proteins were consistent with CREB protein expression among the different groups. The OD values were: PSD95, $0.636 \pm 0.046,0.935 \pm 0.073$ and $0.983 \pm 0.116 ; S Y N, 0.553 \pm 0.034,0.843 \pm 0.107$ and $0.887 \pm 0.089$; and Drebrin, $0.089 \pm 0.014,0.159 \pm 0.015$ and $0.173 \pm 0.018$ in the castration, DHT and P8 groups, respectively (Table II, Fig. 1D-L).

Western blotting. The protein bands for CREB in hippocampus were clearly visible in the different groups (Fig. 2). The integrated OD (IOD) value of CREB in the castration group was $0.589 \pm 0.075$, which was significantly lower compared to the $\mathrm{P} 8$ group at $1.113 \pm 0.152(\mathrm{P}<0.01)$. However, the IOD value for CREB in the DHT group was $1.032 \pm 0.118$, 
Table III. Results of western blotting shows the effects of castration and DHT intervention on the conversion from MCI to Alzheimer's dementia in SAMP8 mice. ${ }^{\mathrm{a}}$

\begin{tabular}{lcccc}
\hline Group & CREB & SYN & PSD95 & Drebrin \\
\hline Castration & $0.589 \pm 0.075$ & $0.453 \pm 0.074$ & $0.109 \pm 0.007$ & $0.189 \pm 0.045$ \\
DHT & $1.032 \pm 0.118^{\mathrm{b}}$ & $0.947 \pm 0.117^{\mathrm{b}}$ & $0.193 \pm 0.018^{\mathrm{b}}$ & $0.632 \pm 0.058^{\mathrm{b}}$ \\
P8 & $1.113 \pm 0.152^{\mathrm{b}}$ & $0.891 \pm 0.126^{\mathrm{b}}$ & $0.205 \pm 0.025^{\mathrm{b}}$ & $0.613 \pm 0.092^{\mathrm{b}}$ \\
\hline
\end{tabular}

${ }^{a}$ Western blotting detected SYN, PSD95, Drebrin, and CREB gene expression in the Castration, DHT and P8 groups. ${ }^{\mathrm{b}} \mathrm{P}<0.01$. DHT, dihydrotestosterone; MCI, mild cognitive impairment; SAMP8, senescence-accelerated mouse prone 8; SYN, synaptophysin; PSD95, postsynaptic density protein 95; CREB, cAMP-response element binding protein; Drebrin, developmentally regulated brain protein.

A

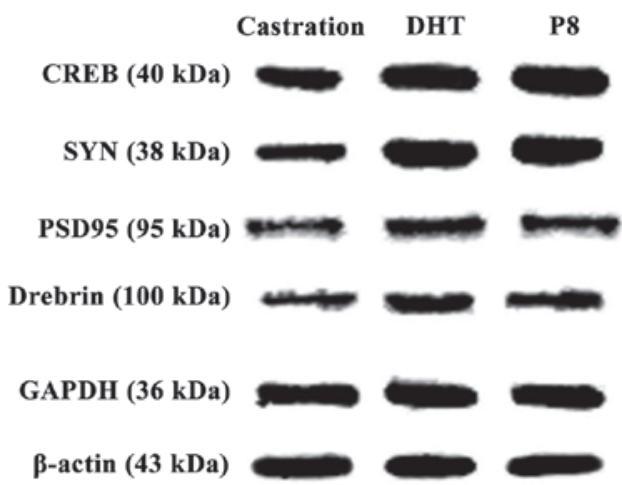

B

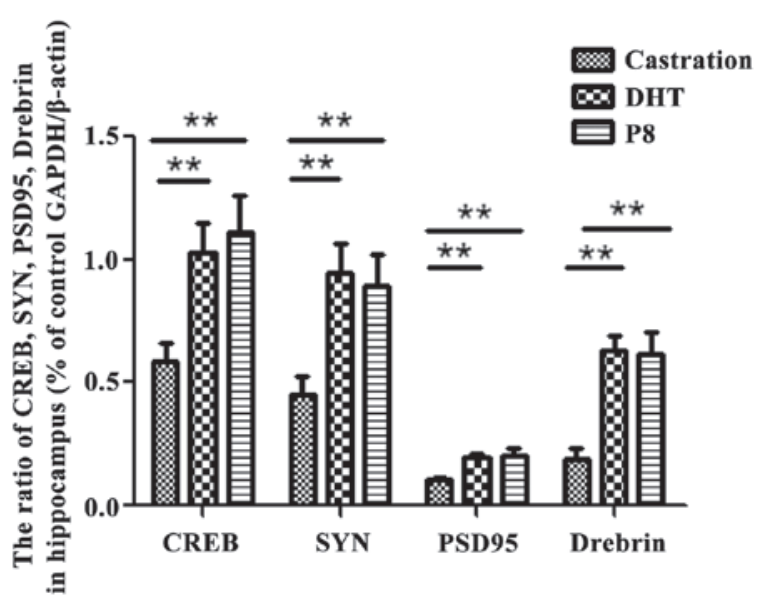

Figure 2. Western blotting showing the effects of castration and DHT intervention on the conversion from MCI to Alzheimer's dementia in SAMP8 mice. Western blot detected SYN, PSD95, Drebrin and CREB protein expression in the (A) castration, DHT and P8 groups. A statistical graph showing the difference of protein expression in the (B) four groups. The results are presented as the mean \pm standard deviation $(n=5)$. Statistical analysis was performed using one-way ANOVA with LSD post-hoc test. ${ }^{* *} \mathrm{P}<0.01$. DHT, dihydrotestosterone; SAMP8, senescence-accelerated mouse prone 8; SYN, synaptophysin; PSD95, postsynaptic density protein 95; CREB, cAMP-response element binding protein; Drebrin, developmentally regulated brain protein.

which was significantly different compared to the castration group $(\mathrm{P}<0.01)$. The expression levels of SYN $(0.453 \pm 0.074$, $0.947 \pm 0.117,0.891 \pm 0.126)$, PSD95 $(0.109 \pm 0.007,0.193 \pm 0.018$, $0.205 \pm 0.025)$, and Drebrin $(0.189 \pm 0.045,0.632 \pm 0.058$, $0.613 \pm 0.092)$ was consistent with that of CREB protein. There was a statistically significant difference $(\mathrm{P}<0.01)$ between the castration group and P8 groups (Table III).

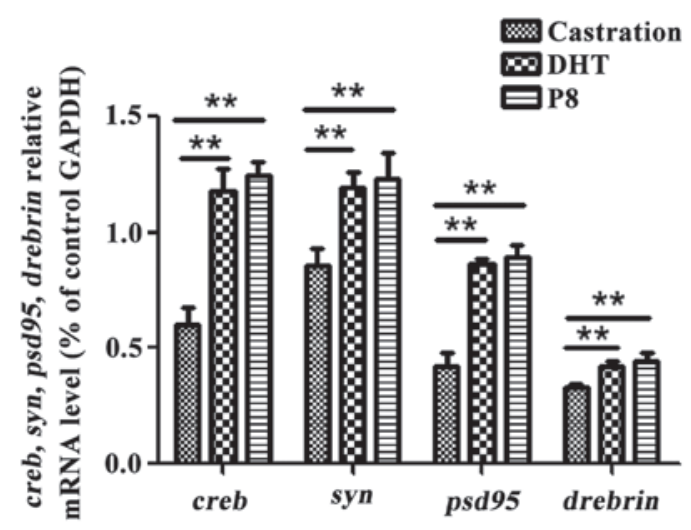

Figure 3. Quantitative polymerase chain reaction showing effects of castration and DHT intervention on the conversion from MCI to Alzheimer's dementia in SAMP8 mice, showing gene expression of SYN, PSD95, Drebrin, and CREB in the Castration, DHT and P8 groups. ${ }^{* *} \mathrm{P}<0.01$. DHT, dihydrotestosterone; SAMP8, senescence-accelerated mouse prone 8; SYN, synaptophysin; PSD95, post synaptic density protein 95; CREB, cAMP-response element binding protein; Drebrin, developmentally regulated brain protein.

$q P C R$. The mRNA expression of creb,psd95, syn and drebrin in hippocampal neurons in different groups was similar to that of their protein expression levels (Fig. 3). The creb, psd95, syn and drebrin mRNAs levels in the castration group were the lowest $(0.597 \pm 0.075,0.853 \pm 0.079,0.420 \pm 0.057$ and $0.328 \pm 0.019$, respectively) and were significantly decreased compared with the $\mathrm{P} 8$ group $(1.245 \pm 0.059, \mathrm{P}<0.01 ; 1.231 \pm 0.116, \mathrm{P}<0.01$; $0.894 \pm 0.052, \mathrm{P}<0.01 ; 0.452 \pm 0.041, \mathrm{P}<0.01$, respectively) and the DHT group $(1.177 \pm 0.101, \mathrm{P}<0.01 ; 1.194 \pm 0.064, \mathrm{P}<0.01$; $0.861 \pm 0.026, \mathrm{P}<0.01 ; 0.422 \pm 0.018, \mathrm{P}<0.01$, respectively) (Table IV, Fig. 3).

\section{Discussion}

The brain is an androgen-sensitive tissue and is influenced by age-related androgen deficiency (12-14). Research on testosterone and its metabolite DHT in rodent and human brains has indicated that reduced brain function is closely related to androgen loss. In particular, some research suggests that sex hormone loss occurs prior to the occurrence of $\mathrm{AD}$, and can be regarded as a cause of $\mathrm{AD}$, rather than the result of $\mathrm{AD}$. This fact suggests that androgen depletion is a precursor event that changes AD clinical manifestation and pathology, which 
Table IV. Results of quantitative polymerase chain reaction (qPCR) display the effects of castration and DHT intervention on the conversion from MCI to Alzheimer's dementia in SAMP8 mice. ${ }^{a}$

\begin{tabular}{lcccc}
\hline Group & creb & syn & psd95 & drebrin \\
\hline Castration & $0.597 \pm 0.075$ & $0.853 \pm 0.079$ & $0.420 \pm 0.057$ & $0.328 \pm 0.019$ \\
DHT & $1.177 \pm 0.101^{\mathrm{b}}$ & $1.194 \pm 0.064^{\mathrm{b}}$ & $0.861 \pm 0.026^{\mathrm{b}}$ & $0.422 \pm 0.018^{\mathrm{b}}$ \\
P8 & $1.245 \pm 0.059^{\mathrm{b}}$ & $1.231 \pm 0.116^{\mathrm{b}}$ & $0.894 \pm 0.052^{\mathrm{b}}$ & $0.452 \pm 0.041^{\mathrm{b}}$ \\
\hline
\end{tabular}

${ }^{\mathrm{a}} \mathrm{qPCR}$ detected syn, psd95, drebrin, and creb gene expression in the Castration, DHT and P8 groups. ${ }^{\mathrm{b}} \mathrm{P}<0.01$. DHT, dihydrotestosterone; MCI, mild cognitive impairment; SAMP8, senescence-accelerated mouse prone 8; syn, synaptophysin; $p s d 95$, postsynaptic density protein 95 ; creb, cAMP-response element binding protein; Drebrin, developmentally regulated brain protein.

may contribute to the understanding of the progression of the disease (40). Despite intensive laboratory and clinical research over the past three decades, effective treatment to delay the onset and progression of $\mathrm{AD}$ is not available. The repetitive failures of clinical trials targeting mild to moderate AD suggest that MCI is a potential way to treat $\mathrm{AD}(41)$.

Compared to other pathological lesions, in the pathological change of $\mathrm{AD}$, synaptic density changes and cognitive impairment are closely related, indicating the extreme importance of mechanisms of contact obstacles of synapses (42). Previous findings suggest that the hippocampus shows decreases of synaptic plasticity in MCI $(31,33)$. However, whether the protein change in the hippocampus reflects the correlation between changes in the number of synapses and low MCI cognitive level is worthy of further investigation.

In young and middle-aged castrated rats, the levels of endogenous androgens were reduced, aggravating pathological changes and hippocampus-related behavior in the animals. Treatment with testosterone or DHT in these castrated rats may significantly reduce pathological damage (43). It seems possible to develop an effective hormone or hormone mimic therapy for prevention of the conversion from MCI to AD (44). Previous findings have indicated that there is close relationship between androgens and synapses $(31,32)$. Experimentally, androgens can reverse the reduced density of hippocampus dendritic spines in castrated male rats, and at the same time an androgen antagonist may significantly weaken this effect (45-47). Those reports suggested that androgens play an important role in maintaining the density of dendritic spine synapses in the hippocampus of normal male animals. Therefore, we hypothesize that the synaptic plasticity of hippocampal structures is correlated with the cognitive function. Studies have reported the existence of a pathway for androgen regulation of synaptic function that is distinct from estrogens; however, the mechanism of this independent androgen pathway was not previously evident (48).

Our previous studies $(31,33)$ have shown that, compared with the control group, the cognitive function of castrated MCI and SAMP8 mice during AD period was impaired. Additionally, DHT improves the aging mice hippocampus-dependent spatial cognitive ability, and castration increases learning and memory disorders. These functions may be associated with changes in the hippocampal formation and morphology of MCI SAMP8 mice. From this perspective, hippocampal neurons and synaptic morphology and structure are worthy of exploration in further experiments. Castration-induced cognitive dysfunction may cause morphological change (32). Castration reduces the thickness of postsynaptic density and postsynaptic PSD, and significantly increases A $\beta$ accumulation and degeneration of organelles in cytoplasm. It is thought that synaptic loss and cognitive decline in $\mathrm{AD}$ are closely related, and senile plaque pathology and partial loss of synapses and deduction of dendritic spines are related (49). Conversely, cognitive disorders, especially in executive function and negative symptoms are considered to be associated with defects of neural plasticity (50). These views explain the correlation between castration-induced cognitive decline and morphological changes.

Our previous results showed that castration aggravates cognitive decline and the loss of synapses and dendritic spines in MCI SAMP8 $(31,33)$. In the process of natural aging, a decrease in testosterone induces the AD pathological change process and decreases synaptic plasticity. Modulation of hippocampal synaptic plasticity by androgens has attracted substantial scientific attention. However, more studies are needed to elucidate the effects of DHT on synaptic plasticity in MCI research. Therefore, we investigated the effect of DHT on the modulation of spine structure and proteins related to synaptic plasticity in the MCI SAMP8 mouse hippocampus. In the present study, castration reduced the levels of synaptic protein markers (PSD95, SYN and Drebrin), which coincided with smaller numbers of synapses and thinner PSDs, as observed in our previous results $(31,32)$. This finding demonstrates that cell viability is low and neural remodeling is suppressed during MCI, and worse structural and morphological changes in castration lead to further decline in spatial learning and memory in the P8 mice.

The above data show that DHT is involved in the regulation of learning and memory, suggesting that physiological levels of DHT can regulate hippocampal function and maintain the hippocampal formation of normal male mice. However, the role of DHT in some proteins, receptors, neurotransmitters and messenger molecules associated with structural basis and physiological functions of synaptic plasticity (51), as well as transcription and protein synthesis needed in long-term memory potentiation remain to be investigated (52).

DHT is thought to increase the thickness of postsynaptic density and postsynaptic density of neurons in castrated animals. These studies indicate that androgen therapy may be effective in delaying the deduction of synapses of hippocampal 
neurons and dendritic spines caused by castration. Thus, DHT can also enhance the synaptic plasticity of hippocampal neurons of $\mathrm{AD}$ mice, to maintain the structure and function of synapses. In this experiment, with P8 sham operation mice as the control group, we studied whether DHT was conducive to maintaining hippocampal neurons and hippocampal synaptic plasticity of castrated mice during MCI period. Synaptic plasticity is the neurobiological basis of learning and memory, and can affect cognitive ability by adjusting the hippocampal synaptic structure and function. Based on this, it is recognized that DHT-induced structural changes of neural plasticity improve the morphological basis of cognitive function (53).

We hypothesized that expression changes of differentiated genes among individuals may be associated with the memory-related behavior of aging rodents. Furthermore, mechanisms of DHT regulating hippocampal synaptic plasticity of MCI SAMP8 mice is unclear. Therefore, we focused on the expression of synaptic plasticity markers (SYN, PSD95, Drebrin) in pyramidal cells of hippocampus, and examined the regulatory mechanism and neural basis effect of DHT.

SYN, PSD95 and Drebrin are involved in synaptic formation and reconstruction (54-56). The immunohistochemical results revealed that DHT decreased SYN, PSD95, Drebrin protein levels of castrated SAMP8 mice. SYN involves the release of presynaptic vesicle protein neurotransmitter and a sign of density of synapses. PSD95 is glutamatergic excitatory postsynaptic density, interacting with NMDA receptor subunits, and participating in the information storage procedure (57). Increased PSD95 expression is capable of adjusting the increase of synaptic activity. Drebrin is an F-actin binding protein expressed in excitatory postsynaptic dendritic spines. It affects synaptic plasticity by regulating the synaptic morphogenesis, and reorganizing postsynaptic density and target receptors $(39,58,59)$. Drebrin, a synaptic marker, is used as a marker of synaptic structural changes. Experimental results show that the effect of DHT on synaptic structure is associated with the expression of protein markers SYN, PSD95 and Drebrin $(60,61)$.

Expression of synaptic proteins SYN, PSD95 and Drebrin reflects changes in cognitive behavior, and shows a positive correlation with synaptic plasticity, a result that is consistent with the results of the present study. This also corroborates our previous studies $(31,33)$ where, after DHT treatment, learning and memory of castrated mice were significantly improved, $\mathrm{A} \beta$ levels were reduced, and the number of dendritic spines density increased $(27,62)$. Expression of SYN and Drebrin in the hippocampus of patients with advanced AD decreased. However, in other studies, after comparing patients with no cognitive impairment (NCI), mild cognitive impairment (MCI) and $\mathrm{AD}$, it was identified that, SYN and Drebrin expression in male hippocampus is different. For SYN expression, MCI and AD were reduced compared to NCI, although the differences were not statistically significant, whereas Drebrin in MCI and AD were reduced by approximately $40 \%$ compared to NCI. In the MCI and AD patients, the difference of synaptic marker protein expression indicates that the plasticity of postsynaptic membrane dendritic spines may be damaged selectively. Furthermore, Drebrin may serve as a protein marker predictor for the onset of memory impairment (63).
The role of androgen in hippocampal pyramidal neurons in male rats has been investigated and with regard to the expression of SYN, it was found that castration does not significantly affect the number of pyramidal cells in the hippocampal CA1 area, although the SYN level is reduced (27). The SYN level of castrated rats is increased after DHT supplementation. Similarly, DHT and estradiol SYN expression levels were increased for castrated male and female rodent animals. The data suggested that sex hormones were closely associated with maintaining a normal SYN expression level in the CA1 area of the rodent hippocampus, indicatign AD-like pathological change caused by lack of sex hormone and pathogenesis that is different to humans. A comparison of testosterone and non-aromatic DHT has shown that, testosterone may, without the aid of a local estrogen pathway, directly affect the hippocampus of the androgen receptor.

Previous studies showed that, CREB has a potential therapeutic effect for AD patients (64). In the early stage of AD, CREB was the most important transcription control factor in the transport network, suggesting that, in the early stage of the disease, dysfunction caused by CREB emerges (65). CREB plays an important role in the neuron plasticity of the brain and long-term memory, that is, synaptic transmission and restructuring. In the pathogenesis of the $A \beta$-induced pathological change of $\mathrm{AD}$, mediated by the CREB pathway, $\mathrm{A} \beta$ changes hippocampus-dependent synaptic plasticity alters and reduces memory loss caused by a number of synapses (66). Thus, CREB protein expression is detected to analyze the impact of DHT in AD pathogenesis.

Our results indicate that $\mathrm{CREB}$ protein expression, and the mRNA transcription, protein and expression of PSD95, SYN and Drebrin of castrated SAMP8 mice was decreases. In the present study, CREB protein and mRNA expression of MCI SAMP8 mice was increased after DHT supplementation, as were the synaptic marker protein and mRNA expression. Thus, these data and our results indicate that enhanced synaptic plasticity and cognitive function are closely related to the improved expression of CREB following DHT increase. Cognitive decline and a decrease of DHT levels are consistent, thus, DHT may be involved in the CREB-induced regulation of synaptic plasticity and cognitive function. DHT, a non-aromatized androgen, cannot be converted to estradiol, and thus plays a role through the estrogen pathway, indicating that the androgen pathway plays a role in synaptic plasticity and the cognitive function of SAMP8 male mice $(31,33)$. These results suggest that, DHT promotes the expression of dendritic spine density and constitutive proteins, rather than converse androgens into estrogens and play a role through the estrogen pathway. Thus, our results are useful for understanding the differences of signaling pathways across the brain between different genders. The results are also consistent with our previous results $(31,33)$, and directly or indirectly support that testosterone and DHT can improve the cognitive behavior of castrated rats and mice, eliminating biological markers $(\mathrm{A} \beta)$ and increasing the number and density of Nissl staining dendritic spines and other experimental results.

The present results indicate that, DHT can affect cognitive and synaptic plasticity of SAMP8 mice during MCI period. Previous findings have shown that cognitive competence and neural plasticity of SAMP8 mice during MCI period 
decline (31,33). Synaptic plasticity in the hippocampus structure may be the cause of reduced spatial memory impairment and this function is related to androgen decline in male mice. In turn, we believe that androgens play an important role in maintaining and regulating hippocampal synaptic plasticity structure in aging mice. Androgen maintains synaptic marker protein (SYN, PSD95 and Drebrin) expression and activates CREB gene expression, thereby regulating synaptic plasticity changes in the basic structure of MCI hippocampus and improving cognitive function. It may be the neural basis mechanism of the wholesome effects which androgen applied to synaptic plasticity in hippocampus. Androgen increases synaptic activity, thereby promoting neural proliferation and maintenance of cells in the MCI SAMP8 mouse hippocampus area, and plays a neuro-protective role. However, the protective effect of androgens in neuroplasticity is worthy of further investigation.

In summary, this study was performed in SAMP8 male mice, and it was determined that the role of mechanism of action of androgens in humans requires additional investigation.

\section{Acknowledgements}

The present study was supported by the National Natural Science Foundation of China Grants (nos. 31271191 and 31471145) and the Natural Science Foundation of Hebei Province Grants (no. C2012206128).

\section{References}

1. Albert MS, DeKosky ST, Dickson D, Dubois B, Feldman HH, Fox NC, Gamst A, Holtzman DM, Jagust WJ, Petersen RC, et al: The diagnosis of mild cognitive impairment due to Alzheimer's disease: Recommendations from the National Institute on Aging-Alzheimer's Association workgroups on diagnostic guidelines for Alzheimer's disease. Alzheimers Dement 7: 270-279, 2011.

2. Alzheimer's Association: 2013 Alzheimer's disease facts and figures. Alzheimers Dement 9: 208-245, 2013.

3. Jack CR Jr, Albert MS, Knopman DS, McKhann GM, Sperling RA, Carrillo MC, Thies B and Phelps CH: Introduction to the recommendations from the National Institute on Aging-Alzheimer's Association workgroups on diagnostic guidelines for Alzheimer's disease. Alzheimers Dement 7: 257-262, 2011.

4. Sperling RA, Aisen PS, Beckett LA, Bennett DA, Craft S, Fagan AM, Iwatsubo T, Jack CR Jr, Kaye J, Montine TJ, et al: Toward defining the preclinical stages of Alzheimer's disease: Recommendations from the National Institute on Aging-Alzheimer's Association workgroups on diagnostic guidelines for Alzheimer's disease. Alzheimers Dement 7 : 280-292, 2011

5. DeCarli C: Mild cognitive impairment: Prevalence, prognosis, aetiology, and treatment. Lancet Neurol 2: 15-21, 2003.

6. Petersen RC: Mild cognitive impairment: Current research and clinical implications. Semin Neurol 27: 22-31, 2007.

7. Jack CR Jr, Petersen RC, Xu YC, O'Brien PC, Smith GE, Ivnik RJ, Boeve BF, Waring SC, Tangalos EG and Kokmen E: Prediction of AD with MRI-based hippocampal volume in mild cognitive impairment. Neurology 52: 1397-1403, 1999.

8. Mufson EJ, Chen EY, Cochran EJ, Beckett LA, Bennett DA and Kordower JH: Entorhinal cortex beta-amyloid load in individuals with mild cognitive impairment. Exp Neurol 158: 469-490, 1999.

9. Price JL and Morris JC: Tangles and plaques in nondemented aging and 'preclinical' Alzheimer's disease. Ann Neurol 45 358-368, 1999.

10. Lautenschlager NT, Riemenschneider M, Drzezga A and Kurz AF: Primary degenerative mild cognitive impairment: Study population, clinical, brain imaging and biochemical findings. Dement Geriatr Cogn Disord 12: 379-386, 2001.
11. Hogervorst E, Bandelow S, Combrinck M and Smith AD: Low free testosterone is an independent risk factor for Alzheimer's disease. Exp Gerontol 39: 1633-1639, 2004.

12. Hogervorst E, Combrinck M and Smith AD: Testosterone and gonadotropin levels in men with dementia. Neuro Endocrinol Lett 24: 203-208, 2003.

13. Hogervorst E, Williams J, Budge M, Barnetson L, Combrinck M and Smith AD: Serum total testosterone is lower in men with Alzheimer's disease. Neuro Endocrinol Lett 22: 163-168, 2001.

14. Paoletti AM, Congia S, Lello $S$, Tedde D, Orrù M, Pistis $M$, Pilloni M, Zedda P, Loddo A and Melis GB: Low androgenization index in elderly women and elderly men with Alzheimer's disease. Neurology 62: 301-303, 2004.

15. Watanabe $T$, Koba $S$, Kawamura $M$, Itokawa $M$, Idei $T$, Nakagawa Y, Iguchi T and Katagiri T: Small dense low-density lipoprotein and carotid atherosclerosis in relation to vascular dementia. Metabolism 53: 476-482, 2004.

16. Manly JJ, Merchant CA, Jacobs DM, Small SA, Bell K, Ferin M and Mayeux R: Endogenous estrogen levels and Alzheimer's disease among postmenopausal women. Neurology 54: 833-837, 2000.

17. Moffat SD, Zonderman AB, Metter EJ, Blackman MR, Harman SM and Resnick SM: Longitudinal assessment of serum free testosterone concentration predicts memory performance and cognitive status in elderly men. J Clin Endocrinol Metab 87: 5001-5007, 2002.

18. Tsolaki M, Grammaticos P, Karanasou C, Balaris V, Kapoukranidou D, Kalpidis I, Petsanis K and Dedousi E: Serum estradiol, progesterone, testosterone, FSH and LH levels in postmenopausal women with Alzheimer's dementia. Hell J Nucl Med 8: 39-42, 2005.

19. Chu LW, Tam S, Wong RL, Yik PY, Song Y, Cheung BM, Morley JE and Lam KS: Bioavailable testosterone predicts a lower risk of Alzheimer's disease in older men. J Alzheimers Dis 21: 1335-1345, 2010.

20. Pike CJ, Rosario ER and Nguyen TV: Androgens, aging, and Alzheimer's disease. Endocrine 29: 233-241, 2006.

21. Liu L, Orozco IJ,PlanelE, Wen Y, Bretteville A, Krishnamurthy P, Wang L, Herman M, Figueroa H, Yu WH, et al: A transgenic rat that develops Alzheimer's disease-like amyloid pathology, deficits in synaptic plasticity and cognitive impairment. Neurobiol Dis 31: 46-57, 2008.

22. Corbyn Z: New set of Alzheimer's trials focus on prevention. Lancet 381: 614-615, 2013.

23. Ambati J and Fowler BJ: Mechanisms of age-related macular degeneration. Neuron 75: 26-39, 2012.

24. Bisaz R, Boadas-Vaello P, Genoux D and Sandi C: Age-related cognitive impairments in mice with a conditional ablation of the neural cell adhesion molecule. Learn Mem 20: 183-193, 2013.

25. Woolley CS and McEwen BS; WoolleyCS: Estradiol mediates fluctuation in hippocampal synapse density during the estrous cycle in the adult rat. J Neurosci 12: 2549-2554, 1992.

26. Scheff SW and Price DA: Synaptic density in the inner molecular layer of the hippocampal dentate gyrus in Alzheimer disease. J Neuropathol Exp Neurol 57: 1146-1153, 1998.

27. Leranth C, Petnehazy O and MacLusky NJ: Gonadal hormones affect spine synaptic density in the CA1 hippocampal subfield of male rats. J Neurosci 23: 1588-1592, 2003.

28. Takeda T, Hosokawa M and Higuchi K: Senescence-accelerated mouse (SAM): A novel murine model of senescence. Exp Gerontol 32: 105-109, 1997.

29. Tresguerres JA, Kireev R, Forman K, Cuesta S, Tresguerres AF and Vara E: Effect of chronic melatonin administration on several physiological parameters from old Wistar rats and SAMP8 mice. Curr Aging Sci 5: 242-253, 2012.

30. Morley JE, Farr SA, Kumar VB and Armbrecht HJ: The SAMP8 mouse: A model to develop therapeutic interventions for Alzheimer's disease. Curr Pharm Des 18: 1123-1130, 2012.

31. Kang L, Li S, Xing Z, Li J, Su Y, Fan P, Wang L and Cui H: Dihydrotestosterone treatment delays the conversion from mild cognitive impairment to Alzheimer's disease in SAMP8 mice. Horm Behav 65: 505-515, 2014.

32. Jia J, Kang L, Li S, Geng D, Fan P, Wang L and Cui H: Amelioratory effects of testosterone treatment on cognitive performance deficits induced by soluble A $\beta 1-42$ oligomers injected into the hippocampus. Horm Behav 64 :477-486, 2013.

33. Li S, Kang L, Zhang C, Xie G, Li N, Zhang Y, Du J and Cui H: Effects of dihydrotestosterone on synaptic plasticity of hippocampus in male SAMP8 mice. Exp Gerontol 48: 778-785, 2013. 
34. Ma J, Zhang Z, Su Y, Kang L, Geng D, Wang Y, Luan F, Wang M and Cui H: Magnetic stimulation modulates structural synaptic plasticity and regulates BDNF-TrkB signal pathway in cultured hippocampal neurons. Neurochem Int 62: 84-91, 2013.

35. Mufson EJ, Binder L, Counts SE, DeKosky ST, de Toledo-Morrell L, Ginsberg SD, Ikonomovic MD, Perez SE and Scheff SW: Mild cognitive impairment: Pathology and mechanisms. Acta Neuropathol 123: 13-30, 2012.

36. Terakawa Y, Agnihotri S, Golbourn B, Nadi M, Sabha N, Smith CA, Croul SE and Rutka JT: The role of drebrin in glioma migration and invasion. Exp Cell Res 319: 517-528, 2013.

37. Tu JC, Xiao B, Naisbitt $\mathrm{S}$, et al: Coupling of mGluR/Homer and PSD-95 complexes by the Shank family of postsynaptic density proteins. Neuron 23: 583-592, 1999.

38. Du H, Guo L, Yan S, Sosunov AA, McKhann GM and Yan SS Early deficits in synaptic mitochondria in an Alzheimer's disease mouse model. Proc Natl Acad Sci USA 107: 18670-18675, 2010.

39. Hatanaka Y, Mukai H, Mitsuhashi K, Hojo Y, Murakami G, Komatsuzaki Y, Sato R and Kawato S: Androgen rapidly increases dendritic thorns of CA3 neurons in male rat hippocampus. Biochem Biophys Res Commun 381: 728-732, 2009.

40. Moffat SD, Zonderman AB, Metter EJ, Kawas C, Blackman MR, Harman SM and Resnick SM: Free testosterone and risk for Alzheimer disease in older men. Neurology 62: 188-193, 2004.

41. Selkoe DJ: Preventing Alzheimer's disease. Science 337 1488-1492, 2012

42. Bell KF and Claudio Cuello A: Altered synaptic function in Alzheimer's disease. Eur J Pharmacol 545: 11-21, 2006.

43. Bilkei-Gorzo A: Genetic mouse models of brain ageing and Alzheimer's disease. Pharmacol Ther 142: 244-257, 2014

44. Demartini DR, Schilling LP, da Costa JC and Carlini CR Alzheimer's and Parkinson's diseases: An environmental proteomic point of view. J Proteomics 104: 24-36, 2014.

45. Balthazart J, Baillien M, Charlier TD and Ball GF: Calcium-dependent phosphorylation processes control brain aromatase in quail. Eur J Neurosci 17: 1591-1606, 2003.

46. Takeuchi Y, Miyamoto E and Fukunaga K: Activation of the rat dopamine D2 receptor promoter by mitogen-activated protein kinase and $\mathrm{Ca} 2+/$ calmodulin-dependent protein kinase II pathways. J Neurochem 83: 784-796, 2002

47. Ishida A, Shigeri Y, Taniguchi T and Kameshita I: Protein phosphatases that regulate multifunctional $\mathrm{Ca} 2+/$ calmodulin-dependent protein kinases: From biochemistry to pharmacology. Pharmacol Ther 100: 291-305, 2003.

48. Ooishi Y, Kawato S, Hojo Y, Hatanaka Y, Higo S, Murakami G, Komatsuzaki Y, Ogiue-Ikeda M, Kimoto T and Mukai H: Modulation of synaptic plasticity in the hippocampus by hippocampus-derived estrogen and androgen. J Steroid Biochem Mol Biol 131: 37-51, 2012.

49. Spires TL, Meyer-Luehmann M, Stern EA, McLean PJ, Skoch J, Nguyen PT, Bacskai BJ and Hyman BT: Dendritic spine abnormalities in amyloid precursor protein transgenic mice demonstrated by gene transfer and intravital multiphoton microscopy. J Neurosci 25: 7278-7287, 2005.

50. Voineskos D, Rogasch NC, Rajji TK, Fitzgerald PB and Daskalakis ZJ: A review of evidence linking disrupted neural plasticity to schizophrenia. Can J Psychiatry 58: 86-92, 2013.

51. Sheng M and Kim MJ: Postsynaptic signaling and plasticity mechanisms 298: 776-780, 2002.
52. Hernandez PJ and Abel T: The role of protein synthesis in memory consolidation: Progress amid decades of debate. Neurobiol Learn Mem 89: 293-311, 2008

53. Hasan A, Nitsche MA, Rein B, Schneider-Axmann T, Guse B, Gruber O, Falkai P and Wobrock T: Dysfunctional long-term potentiation-like plasticity in schizophrenia revealed by transcranial direct current stimulation. Behav Brain Res 224: 15-22, 2011.

54. Honer WG, Dickson DW, Gleeson J and Davies P: Regional synaptic pathology in Alzheimer's disease. Neurobiol Aging 13: 375-382, 1992.

55. Davidsson P and Blennow K: Neurochemical dissection of synaptic pathology in Alzheimer's disease. Int Psychogeriatr 10: $11-23,1998$

56. Harigaya Y, Shoji M, Shirao T and Hirai S: Disappearance of actin-binding protein, drebrin, from hippocampal synapses in Alzheimer's disease. J Neurosci Res 43: 87-92, 1996.

57. Gardoni F, Marcello E and Di Luca M: Postsynaptic density-membrane associated guanylate kinase proteins (PSDMAGUKs) and their role in CNS disorders. Neuroscience 158: 324-333, 2009

58. Kirchberg BC, Cohen JR, Adelsky MB, Buthorn JJ, Gomar JJ, Gordon M, Koppel J, Christen E, Conejero-Goldberg C, Davies P, et al: Semantic distance abnormalities in mild cognitive impairment: Their nature and relationship to function. Am J Psychiatry 169: 1275-1283, 2012.

59. Verma $M$ and Howard RJ: Semantic memory and language dysfunction in early Alzheimer's disease: A review. Int J Geriatr Psychiatry 27: 1209-1217, 2012.

60. Kwon SE and Chapman ER: Synaptophysin regulates the kinetics of synaptic vesicle endocytosis in central neurons. Neuron 70: 847-854, 2011.

61. Sultana R, Banks WA and Butterfield DA: Decreased levels of PSD95 and two associated proteins and increased levels of $\mathrm{BCl} 2$ and caspase 3 in hippocampus from subjects with amnestic mild cognitive impairment: Insights into their potential roles for loss of synapses and memory, accumulation of Abeta, and neurodegeneration in a prodromal stage of Alzheimer's disease. J Neurosci Res 88: 469-477, 2010.

62. Leranth C, Hajszan T and MacLusky NJ: Androgens increase spine synapse density in the CA1 hippocampal subfield of ovariectomized female rats. J Neurosci 24: 495-499, 2004.

63. Counts SE, He B, Nadeem M, Wuu J, Scheff SW and Mufson EJ: Hippocampal drebrin loss in mild cognitive impairment. Neurodegener Dis 10: 216-219, 2012

64. Pugazhenthi S, Wang M, Pham S, Sze CI and Eckman CB: Downregulation of CREB expression in Alzheimer's brain and in $A \beta$-treated rat hippocampal neurons. Mol Neurodegener 6: 60, 2011.

65. Satoh J, Tabunoki H and Arima K: Molecular network analysis suggests aberrant CREB-mediated gene regulation in the Alzheimer disease hippocampus. Dis Markers 27: 239-252, 2009.

66. Saura CA and Valero J: The role of CREB signaling in Alzheimer's disease and other cognitive disorders. Rev Neurosci 22: 153-169, 2011. 